\title{
Entangled massive mechanical oscillators
}

\author{
C. F. Ockeloen-Korppi, ${ }^{1}$ E. Damskägg, ${ }^{1}$ J.-M. Pirkkalainen,${ }^{1}$ A. \\ A. Clerk, ${ }^{2}$ F. Massel, ${ }^{3}$ M. J. Woolley, ${ }^{4}$ and M. A. Sillanpää ${ }^{1, *}$ \\ ${ }^{1}$ Department of Applied Physics, Aalto University, P.O. Box 15100, FI-00076 AALTO, Finland \\ ${ }^{2}$ Institute for Molecular Engineering, University of Chicago, Chicago, IL, 60631, USA \\ ${ }^{3}$ Department of Physics and Nanoscience Center, University of Jyväskylä, \\ P.O. Box 35 (YFL), FI-40014 University of Jyväskylä, Finland \\ ${ }^{4}$ School of Engineering and Information Technology, UNSW Canberra, ACT, 2600, Australia
}

\begin{abstract}
An entangled quantum state of two or more particles or objects exhibits some of the most peculiar features of quantum mechanics. Entangled systems cannot be described independently of each other even though they may have an arbitrarily large spatial separation. Reconciling this property with the inherent uncertainty in quantum states is at the heart of some of the most famous debates in the development of quantum theory [1]. Nonetheless, entanglement nowadays has a solid theoretical and experimental foundation, and it is the crucial resource behind many emerging quantum technologies. Entanglement has been demonstrated for microscopic systems, such as with photons [2 [5], ions [6], and electron spins [7], and more recently in microwave and electromechanical devices [8-10]. For macroscopic objects [11-14], however, entanglement becomes exceedingly fragile towards environmental disturbances. A major outstanding goal has been to create and verify the entanglement between the motional states of slowly-moving massive objects. Here, we carry out such an experimental demonstration, with the moving bodies realized as two micromechanical oscillators coupled to a microwave-frequency electromagnetic cavity that is used to create and stabilise the entanglement of the centre-of-mass motion of the oscillators [15-17. We infer the existence of entanglement in the steady state by combining measurement of correlated mechanical fluctuations with an analysis of the microwaves emitted from the cavity. Our work qualitatively extends the range of entangled physical systems, with implications in quantum information processing, precision measurement, and tests of the limits of quantum mechanics.
\end{abstract}

There exist several proposals for how cavity optomechanical setups could be used to entangle the motional quantum states of two massive mechanical oscillators, see e.g., Refs. [15, 18 22. In such setups, two movable mirrors are incorporated into a resonant optical cavity, and radiation pressure forces inside the cavity can be tailored such that the motion of the mirrors becomes highly correlated and even entangled. This approach does not require any direct interaction between the moving masses, allowing them to be spatially separated. A correlated reduction of noise below the thermal level in the motion of two mechanical oscillators have previously been demonstrated in experiment [23, 24, but not near the quantum level as required for entanglement.

Our work is based on a series of proposals [15, 20, 21. for using reservoir engineering to stabilise two cavitycoupled mechanical oscillators into a steady state that is entangled. The recipes are extensions of an approach used in recent work to squeeze the motion of a single oscillator 25 27. An oscillator with frequency $\omega_{1}$ and position operator $x_{1}(t)=X_{1}(t) \cos \omega_{1} t+P_{1}(t) \sin \omega_{1} t$, is squeezed if the variance of either quadrature amplitude operators $X_{1}$ or $P_{1}$ is smaller than the quantum zeropoint fluctuation level. Introducing a second oscillator with frequency $\omega_{2}$ and position operator $X_{2}$ expressed in terms of quadratures as above, one can introduce four collective quadrature operators, $X_{ \pm}=\frac{1}{\sqrt{2}}\left(X_{2} \pm X_{1}\right)$ and $P_{ \pm}=\frac{1}{\sqrt{2}}\left(P_{2} \pm P_{1}\right)$. The state corresponding to either the variances of $X_{+}$and $P_{-}$, or $X_{-}$and $P_{+}$, being reduced below the quantum zero-point fluctuations level is a canonical entangled state known as the two-mode squeezed state. Such a state leads to violations of local realism, and is analogous to the state considered in the EPR paradox [1, see Fig. 1 1 . Specifically, the state is entangled if $\left\langle X_{+}^{2}\right\rangle+\left\langle P_{-}^{2}\right\rangle<1$, a criterion commonly referred to as the Duan inequality [28].

In our experiment, a single driven cavity mode is used both to prepare a correlated state of two mechanical oscillators as well as to directly measure fluctuations in the $X_{+}$collective quadrature (via a two-mode BAE measurement) [15 17]; we find $\left\langle X_{+}^{2}\right\rangle \simeq 0.41 \pm 0.04$. While a direct measurement of $P_{-}$is not possible, by constraining system parameters and analyzing the full output spectrum of our cavity, we are also able to infer its fluctuations, $\left\langle P_{-}^{2}\right\rangle \simeq 0.42 \pm 0.08$ (see e.g. Ref. [25]). We hence have an entangled state with $\left\langle X_{+}^{2}\right\rangle+\left\langle P_{-}^{2}\right\rangle \simeq 0.83 \pm 0.13<1$ of two slowly moving massive oscillators each consisting of approximately $10^{12}$ atoms.

As shown in Fig. 1 1 , we use a microwave-frequency realization of cavity optomechanics involving two micromechanical drum oscillators [29, and a superconducting on-chip circuit acting as the electromagnetic cavity 

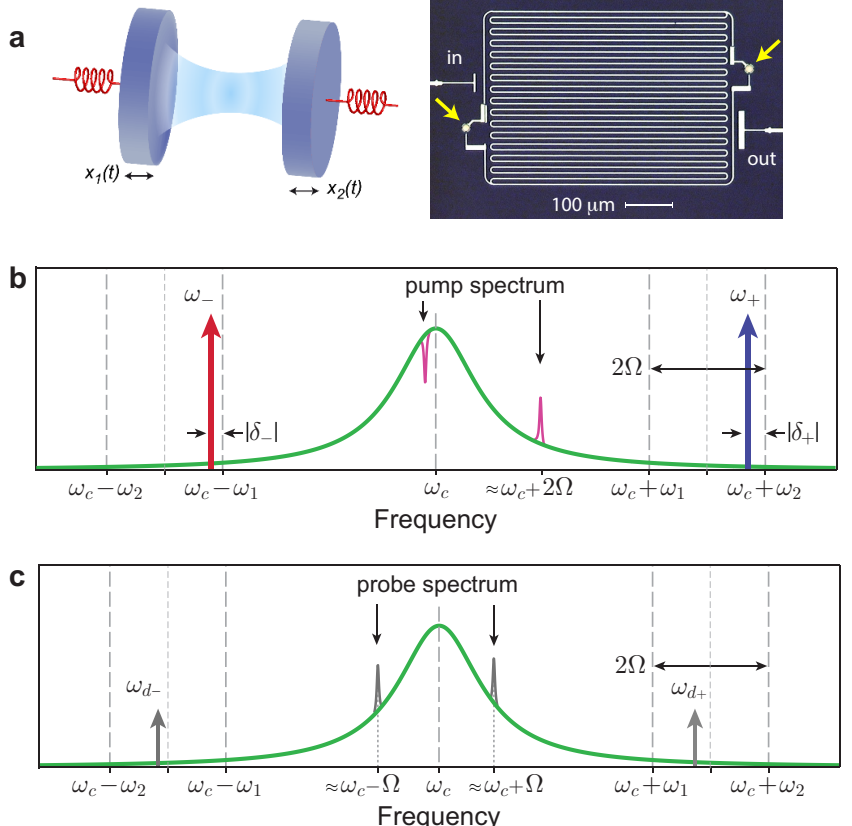

d

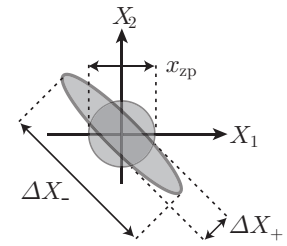

FIG. 1. Creating and detecting motional entanglement. a, Schematic of two vibrating mirrors coupled via an electromagnetic cavity (left), and a micrograph showing the microwave optomechanical device consisting of a superconducting transmission line resonator, whose opposite ends are connected by two mechanical drum-type oscillators marked with arrows (right). The device is fabricated on a quartz chip out of aluminium. b, Spectral picture of the pump microwave frequencies (see text) applied about the cavity spectrum. c, Two additional weak probe tones are applied in order to reconstruct the $X_{+}$collective mechanical quadrature using a two-mode back-action evading measurement. d, Illustration of the correlations in two-mode squeezing in terms of fluctuations (shaded) of the quadrature amplitudes. Left, the sum of $X$ quadratures of the two oscillators fluctuates less than the zero-point level (circle). Right, the difference of $P$ quadratures is similarly localized.

(frequency $\omega_{\mathrm{c}}$ ). The oscillators' positions affect the total capacitance, and hence modulate the frequency of the cavity. This creates an effective radiation pressure interaction as with optical cavities and mirrors. In order to generate two-mode squeezing and entanglement, we pump the system with two strong pump microwave tones at the frequencies $\omega_{-}$and $\omega_{+}$, below and above (respectively) the cavity frequency as shown in Fig. 1 1 b.

To describe this system, we initially assume for simplicity that the two oscillators have equal single-photon radiation-pressure coupling strengths $g_{0}$, and that the

pumps are applied at the red and blue sideband frequencies $\omega_{-}=\omega_{c}-\omega_{1}$ and $\omega_{+}=\omega_{c}+\omega_{2}$, respectively. Details of the derivations, including non-idealities, are discussed in the supplement 30. The strong pumps enhance the radiation-pressure interaction, yielding manyphoton coupling rates $G_{ \pm}=g_{0} \alpha_{ \pm}$, where $\alpha_{ \pm}$are the field amplitudes induced in the resonator due to the pump tones at $\omega_{ \pm}$. We also introduce the mechanical Bogoliubov modes which are obtained by a two-mode squeezing transformation on the original mechanical annihilation operators, viz. $\beta_{1}=b_{1} \cosh r+b_{2}^{\dagger} \sinh r$, and $\beta_{2}=b_{2} \cosh r+b_{1}^{\dagger} \sinh r$, where $\tanh r=G_{+} / G_{-}$. Defining $\Omega=\left(\omega_{2}-\omega_{1}\right) / 2$, and working in a rotating frame (at $\omega_{\mathrm{c}}+\Omega$ for the cavity, $\left(\omega_{2}+\omega_{1}\right) / 2$ for each mechanical oscillator), the linearized optomechanical Hamiltonian is:

$$
\begin{aligned}
H= & -\Omega a^{\dagger} a+\Omega\left(\beta_{2}^{\dagger} \beta_{2}-\beta_{1}^{\dagger} \beta_{1}\right) \\
& +\mathcal{G}\left[a^{\dagger}\left(\beta_{1}+\beta_{2}\right)+a\left(\beta_{1}^{\dagger}+\beta_{2}^{\dagger}\right)\right] .
\end{aligned}
$$

Here $\mathcal{G}=\sqrt{G_{-}^{2}-G_{+}^{2}}$ is an effective optomechanical coupling rate. This Hamiltonian is essentially that in Ref. 15, but with the pump tones set as in Ref. [21. It describes cooling of the Bogoliubov modes by cavity cooling towards their ground state, which corresponds to a stabilised, two-mode squeezed state of the bipartite mechanical system. In contrast to dynamical protocols, e.g. Refs. [10, 13, the system hence stays entangled indefinitely. Here, non-degenerate mechanical frequencies are essential, so that both Bogoliubov modes are efficiently cooled by different frequency components of the cavity.

Although the pumping frequencies correspond to those in Ref. 21], we stress that in our scheme both pumps necessarily couple to both oscillators as in [15, entailing that the rotating-wave approximation involves only a modest requirement that the cavity linewidth $\kappa \ll \omega_{1,2}$, as is well satisfied in the experiment. Note that we will work with $\Omega \lesssim \kappa$, so that both $\beta_{1}$ and $\beta_{2}$ have appreciable coupling to the cavity via Eq. (1). If there are asymmetries in the single-photon couplings $g_{1}$ and $g_{2}$ for the two oscillators, or if the pump tones are detuned (by the amounts $\delta_{ \pm}$, see Fig. 1 $\mathrm{b}$, where we also define $\left.\Delta=\left(\delta_{+}-\delta_{-}\right) / 2\right)$ from the mechanical sidebands, there are additional terms in the Hamiltonian Eq. (1), and one might expect that the steady-state entanglement is reduced (see [30] for the full model). However, we find numerically that one can greatly compensate for asymmetries in couplings by optimising the pump detunings $\delta_{ \pm} \neq 0$. We find, as well, that with the current set of parameters, it is beneficial to red-pump the lower-frequency oscillator 1 . We will follow these practices in the experiment.

As displayed in Fig. 1k, an essential part of the entanglement verification strategy consists of two-mode backaction evading (BAE) detection [16, 17 operated in the same cavity mode, allowing for mapping the mechanical motion to the output field. This involves two relatively 
weak probe tones applied at $\omega_{\mathrm{d} \pm} \approx \omega_{\mathrm{c}} \pm\left(\omega_{2}+\omega_{1}\right) / 2$, approximately in the middle of the sideband frequencies. In order to preserve the same rotating frame for creation and detection of the two-mode squeezing, we strictly require $\omega_{\mathrm{d}+}-\omega_{\mathrm{d}-}=\omega_{+}-\omega_{-}$, ideally up to complete phase coherence between the tones. In a manner similar to the pumps, the probes induce effective couplings $g_{ \pm}=g_{0} \alpha_{ \pm}^{\mathrm{d}}$, with the amplitudes $\alpha_{ \pm}^{\mathrm{d}}$, which in the ideal two-mode back-action evading case are equal. Since we are using the same cavity mode for both creating the entanglement via the pumps and detecting it, the pump spectra and probe spectra need to be independent. This is achieved by ensuring that the mechanical contributions to the output cavity spectrum from the pump and probe tones have negligible spectral overlap. Hence, the faithful reconstruction of the $X_{+}$collective quadrature spectrum from the probe signal is possible. In contrast to BAE detection of single-mode squeezing [25], both the pumps and probes can be set to optimal frequencies for the creation and detection of two-mode squeezing (see Fig. 1 1 ,c).

In our device the two oscillators are far separated by 600 microns, they have no direct coupling, and the system is well described by Eq. (1). We use the fundamental drum modes of the oscillators with the resonance frequencies $\omega_{1} / 2 \pi \simeq 10.0 \mathrm{MHz}$ and $\omega_{2} / 2 \pi \simeq 11.3 \mathrm{MHz}$, and linewidths $\gamma_{1} / 2 \pi \simeq 106 \mathrm{~Hz}$ and $\gamma_{2} / 2 \pi \simeq 144 \mathrm{~Hz}$, respectively. The microwave cavity, with the frequency $\omega_{\mathrm{c}} / 2 \pi \simeq 5.5 \mathrm{GHz}$, has separate input and output ports. All the input signals are applied through a port coupled weakly at the rate $\kappa_{\mathrm{Ei}} / 2 \pi \simeq 60 \mathrm{kHz}$, whereas the output is strongly coupled at $\kappa_{\mathrm{Eo}} / 2 \pi \simeq 1.13 \mathrm{MHz}$. The cavity also has internal losses at the rate $\kappa_{\mathrm{I}} / 2 \pi \simeq 190 \mathrm{kHz}$, and all the loss channels sum to the total linewidth $\kappa / 2 \pi \simeq 1.38 \mathrm{MHz}$. We find that our fabrication process can produce basically identical single-photon couplings, $g_{1} / g_{2} \simeq 0.98$ for two oscillators of different frequencies, in fact, this is more than sufficient for the purpose of generating entanglement since numerically we find that an asymmetry up to $\sim 20 \%$ can be compensated via detunings.

The motion of the mechanical oscillators is measured via the power scattered from the applied microwave tones, both pumps and probes, by their interaction with the oscillators. We collect this weak signal using standard techniques, including a low-noise cryogenic microwave amplifier followed by room-temperature signal analysis. A sequence of calibrations, described in detail in the supplement [30] is important for the experiment. First, based on a standard thermal calibration using a single red-detuned tone, the mechanical modes are found to thermalize down to the equilibrium phonon occupation numbers $n_{1}^{\mathrm{T}} \simeq 41$ and $n_{2}^{\mathrm{T}} \simeq 30$ for oscillators 1 and 2, respectively, at the base temperature $\simeq 14 \mathrm{mK}$ of the dry dilution refrigerator. These values imply the initial variances of the collective quadratures $\left\langle X_{ \pm}^{2}\right\rangle^{\mathrm{T}}$ and
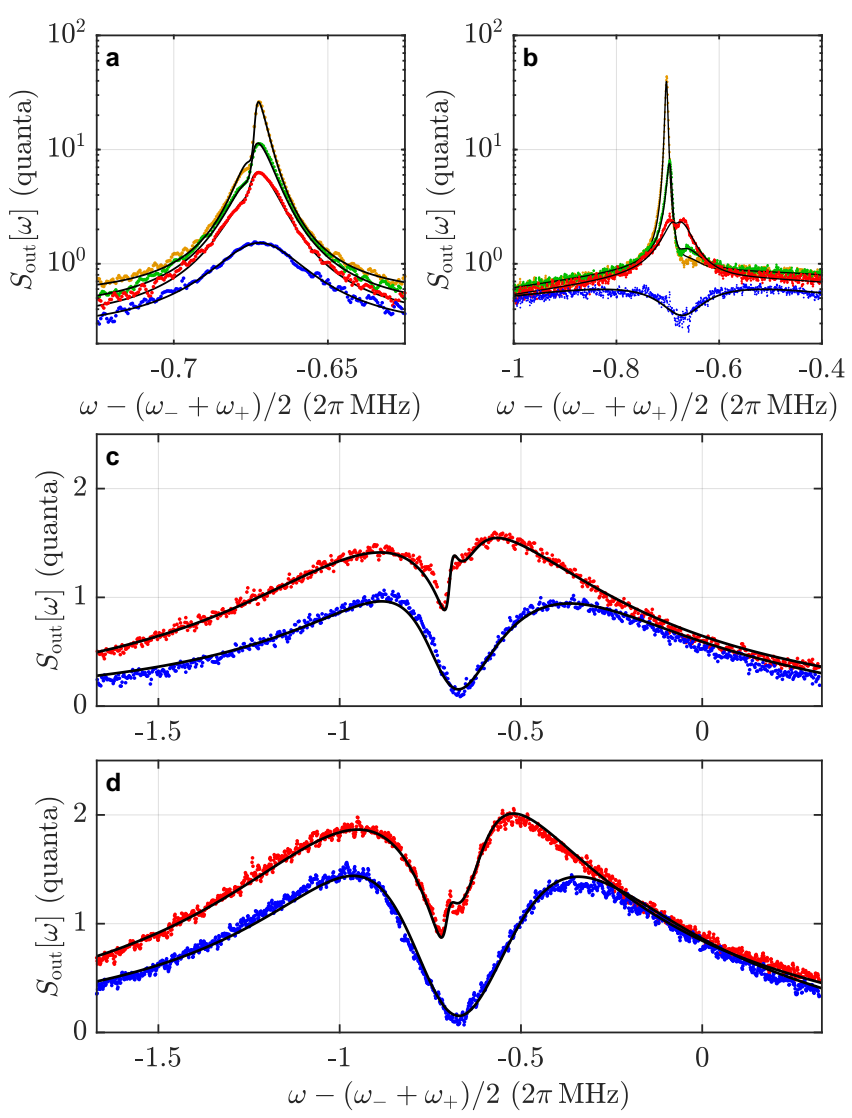

FIG. 2. Pump spectra under two-tone driving. a, The pump amplitudes are as $G_{-} / 2 \pi \simeq 103 \mathrm{kHz}$, and varying $G_{+} / 2 \pi \simeq 82,74,67 \mathrm{kHz}$ from top to bottom. b, $G_{-} / 2 \pi \simeq$ $201 \mathrm{kHz}, G_{+} / 2 \pi \simeq 135 \mathrm{kHz}$, and varying detuning $\Delta / 2 \pi \simeq$ $[19.4,9.6,0.1] \mathrm{kHz}$ from left to right. c, Labelled dataset $C$ in the following, with $G_{-} / 2 \pi \simeq 278 \mathrm{kHz}, G_{+} / 2 \pi \simeq 166 \mathrm{kHz}$. d, Dataset $D$, having higher pump powers $G_{-} / 2 \pi \simeq 332 \mathrm{kHz}$, $G_{+} / 2 \pi \simeq 210 \mathrm{kHz}$. The blue lines, shown for reference, are the sideband cooling calibrations run for oscillator 1 . Theory curves are given by the solid lines. The remaining parameters are listed in the supplementary [30].

$\left\langle P_{ \pm}^{2}\right\rangle^{\mathrm{T}} \simeq 36$

We proceed with standard sideband cooling of each mechanical oscillator separately using a single red-detuned pump. This allows characterization of the behaviour of the system under intense pumping. Importantly, it calibrates the gain of the detection system for the later interpretation of the spectrum under two-tone pumping, as well as the effective coupling of the red-detuned tone. The goal of calibrating the probe tones is to use the total power in the probe spectra as a straightforward thermometer for the quadratures. Similar to the single red tone case, we run a thermal calibration with both probe tones on that allows us to determine the collective occupation number measured at a small probe power. Second, a power sweep calibration of the probes connects a given signal strength to the quadrature variance. 
Next we discuss the main experiment that uses two pairs of tones, namely the pumps and the probes. The pump tones are used to create entanglement, and the probe tones enable tomography of the collective mechanical state. First, we focus on the spectrum due to the pump tones. In Fig. 2, we display the pump output spectra $S_{\text {out }}[\omega]$ from the cavity under several pumping conditions, given in absolute units determined via the gain calibration. This spectrum is one piece of information available for characterizing the entanglement of the two oscillators, since it allows for the inference of the effective temperatures of the three reservoirs. Our theoretical modeling uses standard input-output theory for electromagnetic cavities, treating the pump and probe tones as effectively belonging to independent modes. The variances of the collective quadratures, with a given set of parameters, can be evaluated within the same framework.

Now consider the spectrum resulting from the probe tones. The ability to control the relative phase $\phi$ of the two probe tones allows us to infer the variance of a general collective quadrature $X_{+}^{\phi}=X_{+} \cos \phi+P_{+} \sin \phi$. Written in terms of the spectra $S_{\mathrm{X}_{+}}[\omega]$ and $S_{\mathrm{P}_{+}}[\omega]$ of $X_{+}$ and $P_{+}$, respectively, the measured spectrum is then proportional to $S_{\mathrm{X}_{+}^{\phi}}[\omega]=S_{\mathrm{X}_{+}}[\omega] \cos ^{2} \phi+S_{\mathrm{P}_{+}}[\omega] \sin ^{2} \phi$. In the measurement, the probe signal is visible as peaks on top of the pump spectrum at the frequencies $\sim \omega_{\mathrm{c}} \pm \Omega$ on either side of the cavity (see Fig. 1 c). In Fig. 3 we display the measured probe spectra $S_{\text {out }}^{\mathrm{d}}[\omega]$, having subtracted the background measured in the absence of the probe tones, corresponding to dataset $C$. The probe power at $g_{ \pm} / 2 \pi \simeq 40 \mathrm{kHz}$ was kept much smaller than the pump power. The theoretical model, shown with the same parameters for all curves, is in excellent agreement with the experiment, including the positions and markedly nonLorentzian lineshapes of the peaks, all of which depend on the phase. The unusual shapes physically arise due to the fact that the two oscillators are pumped in a very unequal manner, and they exhibit individual optical springs which add up to the collective spectrum. We infer the quadrature variance $\left\langle\left(X_{+}^{\phi}\right)^{2}\right\rangle$ from the total integrated area of the peaks. This method, as opposed to relying on the particular shapes of the peaks, is insensitive to the phase drift of the microwave sources occurring during the data acquisition. Within a typical integration time for one curve of approximately 30 minutes, $\phi$ can drift several degrees, leading to slight departures from the theoretically determined curves.

From our modeling [30, we confirm that the probe peak area faithfully reproduces the quadrature variances following our calibration as described above. This rigorously holds if the probe powers are perfectly matched, i.e., $\alpha_{+}^{\mathrm{d}}=\alpha_{-}^{\mathrm{d}}$, which was calibrated without the pump tones. However, for the theory fits in Fig. 3, we need to determine an imbalance of $g_{-} / g_{+} \simeq 1.055$, which we attribute to a shift of the cavity frequency when the strong

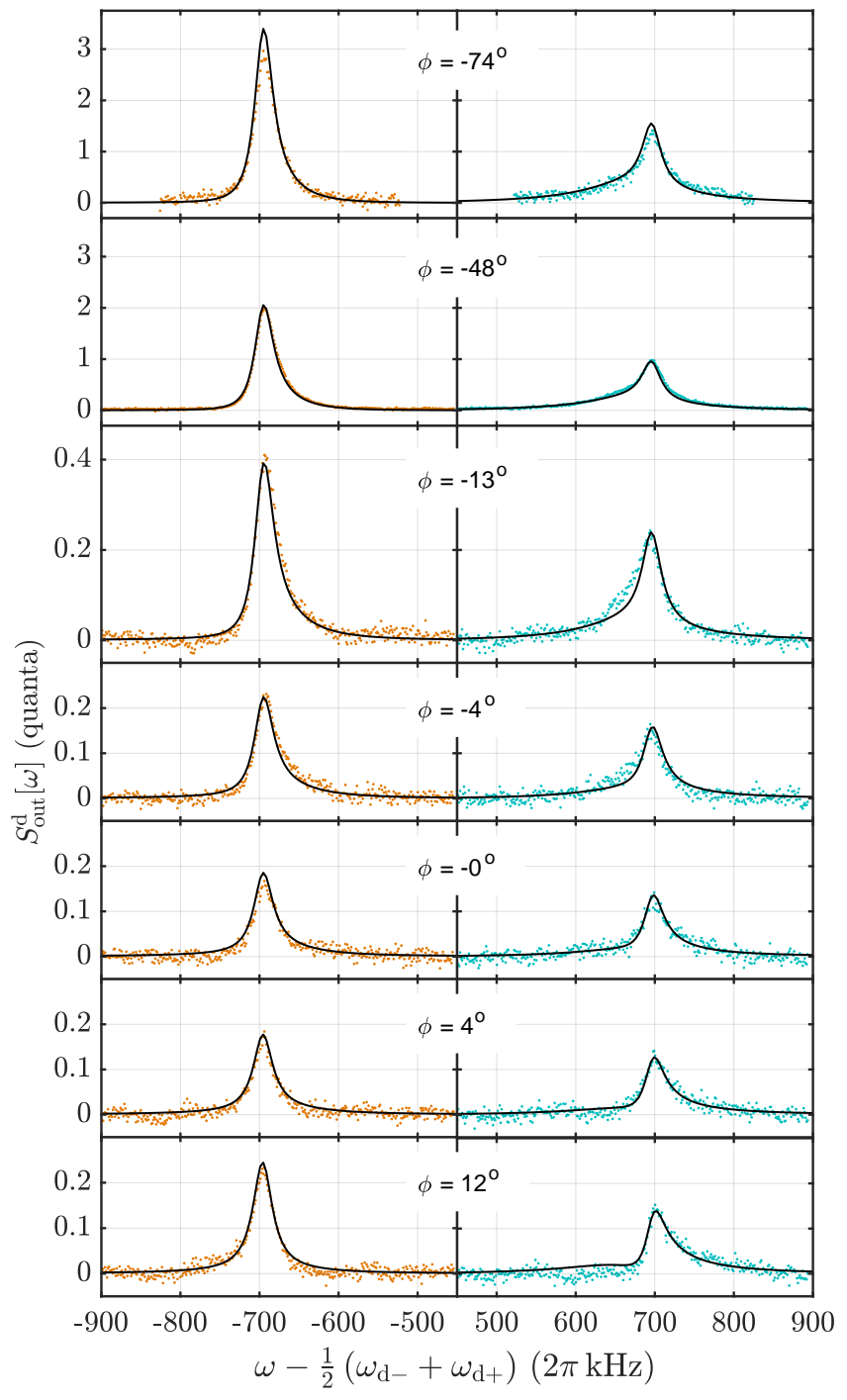

FIG. 3. Two-mode back-action evading readout. The probe output spectrum recorded with the same parameters as the pump spectrum in Fig. 2 (dataset $C$ ), i.e., $G_{-} / 2 \pi \simeq 278 \mathrm{kHz}$, $G_{+} / 2 \pi \simeq 166 \mathrm{kHz}$. The probe phases are written in the panels, and the solid lines are theoretical predictions. The four lowermost panels exhibit two-mode squeezing below the quantum zero-point fluctuation level. The two uppermost panels, having a different vertical scale, present hot quadratures.

pumps are present, causing a reduction of the blue probe tone in the system involving an input filter with a steep slope at the blue side. The reduced $g_{+}$, given that $g_{-}$ stays constant, means that the probe area will underestimate the quadrature variance, here by $\simeq 18 \%$, a number obtained numerically from the model [30]. We will hence scale up the quadrature variances inferred from the probe areas by this percentage. A similarly low blue pump power as compared to the best estimate is observed in the pump spectra as well, but the red pump matches the calibration, supporting the handing of the imbalance as described. We emphasize that the probe inference does 

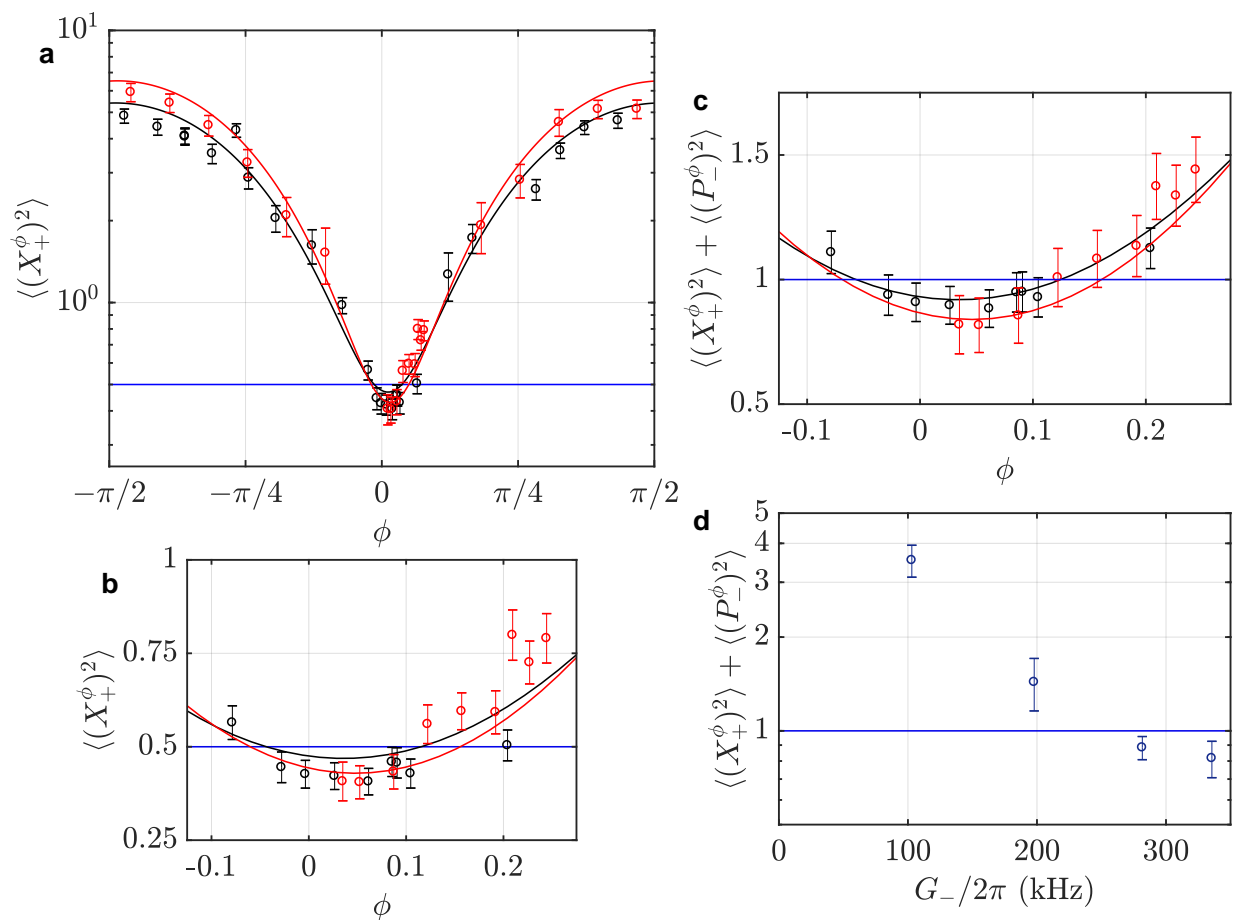

FIG. 4. Fluctuations of collective quadratures. a, The $X_{+}^{\phi}$ quadrature variance measured by the probe spectra. The black circles come from the dataset $C$, and red circles correspond to dataset $D$. $\mathbf{b}$, Zoom-in of a. c, The Duan quantity for entanglement as a function of the probe tone phase. $\mathbf{d}$, The Duan quantity, for the optimal value of $\phi$, as a function of the strength of the red-detuned pump tone. The solid lines coloured similar to the datasets are theoretical fits using the bath temperatures determined by the pump spectra. The blue horizontal line marks the quantum zero-point fluctuations level.

not assume anything about the mechanical oscillators or the dynamics induced by the pumps, but only assumes an understanding of the dynamics associated with the probe tones.

In Fig. 4a,b we display a tomographic measurement of the $X_{+}^{\phi}$ collective quadrature, with $95 \%$ statistical confidence intervals. In the optimal case of $\phi \simeq 4^{\circ}$ minimizing the variance in Fig. 3, we obtain $\left\langle\left(X_{+}^{\phi}\right)^{2}\right\rangle \simeq 0.41 \pm 0.04$; that is $0.9 \mathrm{~dB}$ below vacuum. Several points fall well below the quantum zero-point noise level in both datasets $C$ and $D$. Since the best theoretical fit to the measured probe spectra is obtained with dataset $C$, we base our main claims on this data. We believe that dataset $D$ was subject to larger phase drifts during the data acquisition. Generally, the spectra and responses are highly sensitive to the parameters, and it is remarkable that we reach such good agreement between theory and experiment with a fixed set of parameters describing different measurements (pump, probe, and linear response 30]).

Now consider the measurement of the variance of the $P_{-}$quadrature, needed for examining the Duan criterion and verifying quantum entanglement. The two-mode BAE probe detection, as mentioned, does not couple to $P_{-}$or $X_{-}$. We therefore use the other source of information available, namely the pump spectrum, and then combine this information with that provided by the probe detection. A least-squares fit to an analytical expression describing the pump spectrum, using the three bath temperatures as adjustable parameters, combined with the aforementioned calibrations, allows the evaluation of these variances. The fits are shown in Fig. 2, displaying an excellent agreement to the experiment. For dataset $C$, we obtain the variance $\left\langle P_{-}^{2}\right\rangle \simeq 0.45 \pm 0.08$. For $X_{+}$ quadrature, we similarly get $\left\langle X_{+}^{2}\right\rangle \simeq 0.46 \pm 0.08$, close to the value obtained from the direct $\mathrm{BAE}$ detection method described above (for the confidence intervals, see next section). Given our knowledge of system parameters and the dynamics of this scheme, the two quadratures are expected to have variances within $5 \%$ of one another [30], providing additional evidence for the value of $\left\langle P_{-}^{2}\right\rangle$ based on BAE detection.

The error analysis of the probe measurement uses straightforward error propagation of the experimental calibrations, and of a statistical error from integrating the probe peak area. For the pump spectrum, the analysis is complicated because it involves more parameters, some of which can sensitively affect the steady-state entanglement. Here we adopt an error analysis method known as the Bayesian Monte Carlo method, similar to Ref. [25], to rigorously infer the parameters including uncertainties and correlations. The method generates a sample of the parameter distribution for which the theory model agrees 
with the measured pump spectra within the statistical uncertainty [30. We sample the posterior distributions of all parameters, and use the distributions to estimate the confidence limits of the $P_{-}$quadrature variance. We obtain [30] that at $96 \%$ probability, $\left\langle P_{-}^{2}\right\rangle<0.5$ in case of the data in Fig. 3 (dataset $C$ ). This approach also yields the most likely value $\left\langle P_{-}^{2}\right\rangle \simeq 0.42 \pm 0.08$ that agrees with the values obtained above, but is determined independently.

The best estimate of the Duan quantity is given by combining all the information, namely $\left\langle X_{+}^{2}\right\rangle$ from probe detection, and that for $\left\langle P_{-}^{2}\right\rangle$ as explained above, with equal-weight error bars. We therefore conclude that individually the fluctuations of $X_{+}$and $P_{-}$are below the quantum zero-point fluctuations level, and in particular that when summed, they satisfy the Duan bound for entanglement, $\left\langle X_{+}^{2}\right\rangle+\left\langle P_{-}^{2}\right\rangle<1$ for dataset $C$ at $98 \%$ probability, exceeding the standard $2 \sigma$ confidence. The conclusion is further supported by dataset $D$ (red in Fig. 4) [30, satisfying the Duan bound even more strongly at $>99 \%$ probability.

The entangled mechanical oscillators we have prepared can find practical use in sensitive measurements. Combined with two-mode back-action evading measurements, squeezed optical inputs, and parametric amplification, they would allow for infinitely precise reconstruction of classical forces driving the oscillators. This has implications for gravitational wave detection, and metrology more broadly. Fundamentally, the entanglement of massive mechanical oscillators establishes a new regime for experimental quantum mechanics. The correlations we have created between the motion of two oscillators are so strong that they can be said to share a channel of spooky action, thereby measuring one would influence the other non-locally. In the future one could carry out quantum teleportation of motional states or test Bell inequalities with massive mechanical objects.

* mika.sillanpaa@aalto.fi

[1] Einstein, A., Podolsky, B. \& Rosen, N. Can quantummechanical description of physical reality be considered complete? Phys. Rev. 47, 777 (1935).

[2] Aspect, A., Dalibard, J. \& Roger, G. Experimental Test of Bell's Inequalities Using Time-Varying Analyzers. Phys. Rev. Lett. 49, 1804 (1982).

[3] Heidmann, A. et al. Observation of quantum noise reduction on twin laser beams. Phys. Rev. Lett. 59, 2555-2557 (1987).

[4] Ou, Z. Y., Pereira, S. F., Kimble, H. J. \& Peng, K. C. Realization of the Einstein-Podolsky-Rosen paradox for continuous variables. Phys. Rev. Lett. 68, 3663-3666 (1992).

[5] Bowen, W. P., Treps, N., Schnabel, R. \& Lam, P. K. Experimental demonstration of continuous variable polarization entanglement. Phys. Rev. Lett. 89, 253601 (2002).
[6] Jost, J. D. et al. Entangled mechanical oscillators. Nature 459, 683-685 (2009).

[7] Hensen, B. et al. Loophole-free Bell inequality violation using electron spins separated by 1.3 kilometres. Nature 526, 682-686 (2015).

[8] Steffen, M. et al. Measurement of the entanglement of two superconducting qubits via state tomography. Science 313, 1423-1425 (2006).

[9] DiCarlo, L. et al. Preparation and measurement of threequbit entanglement in a superconducting circuit. Nature 467, 574-578 (2010).

[10] Palomaki, T. A., Teufel, J. D., Simmonds, R. W. \& Lehnert, K. W. Entangling mechanical motion with microwave fields. Science 342, 710-713 (2013).

[11] Leggett, A. J. Macroscopic quantum systems and the quantum theory of measurement. Prog. Theor. Phys. Suppl. 69, 80 (1980).

[12] Julsgaard, B., Kozhekin, A. \& Polzik, E. S. Experimental long-lived entanglement of two macroscopic objects. Nature 413, 400-403 (2001).

[13] Lee, K. C. et al. Entangling macroscopic diamonds at room temperature. Science 334, 1253-1256 (2011).

[14] Klimov, P. V., Falk, A. L., Christle, D. J., Dobrovitski, V. V. \& Awschalom, D. D. Quantum entanglement at ambient conditions in a macroscopic solid-state spin ensemble. Science Advances 1 (2015).

[15] Woolley, M. J. \& Clerk, A. A. Two-mode squeezed states in cavity optomechanics via engineering of a single reservoir. Phys. Rev. A 89, 063805 (2014).

[16] Woolley, M. J. \& Clerk, A. A. Two-mode back-actionevading measurements in cavity optomechanics. Phys. Rev. A 87, 063846 (2013).

[17] Ockeloen-Korppi, C. F. et al. Quantum backaction evading measurement of collective mechanical modes. Phys. Rev. Lett. 117, 140401 (2016).

[18] Mancini, S., Giovannetti, V., Vitali, D. \& Tombesi, P. Entangling macroscopic oscillators exploiting radiation pressure. Phys. Rev. Lett. 88, 120401 (2002).

[19] Pinard, M. et al. Entangling movable mirrors in a doublecavity system. Europhysics Letters 72, 747 (2005).

[20] Tan, H., Li, G. \& Meystre, P. Dissipation-driven twomode mechanical squeezed states in optomechanical systems. Phys. Rev. A 87, 033829 (2013).

[21] Wang, Y.-D. \& Clerk, A. A. Reservoir-engineered entanglement in optomechanical systems. Phys. Rev. Lett. 110, 253601 (2013).

[22] Li, J., Haghighi, I. M., Malossi, N., Zippilli, S. \& Vitali, D. Generation and detection of large and robust entanglement between two different mechanical resonators in cavity optomechanics. New Journal of Physics 17, 103037 (2015).

[23] Mahboob, I., Okamoto, H., Onomitsu, K. \& Yamaguchi, H. Two-mode thermal-noise squeezing in an electromechanical resonator. Phys. Rev. Lett. 113, 167203 (2014).

[24] Pontin, A. et al. Dynamical two-mode squeezing of thermal fluctuations in a cavity optomechanical system. Phys. Rev. Lett. 116, 103601 (2016).

[25] Wollman, E. E. et al. Quantum squeezing of motion in a mechanical resonator. Science 349, 952-955 (2015).

[26] Pirkkalainen, J.-M., Damskägg, E., Brandt, M., Massel, F. \& Sillanpää, M. A. Squeezing of quantum noise of motion in a micromechanical resonator. Phys. Rev. Lett. 115, 243601 (2015).

[27] Lecocq, F., Clark, J. B., Simmonds, R. W., Aumentado, 
J. \& Teufel, J. D. Quantum nondemolition measurement of a nonclassical state of a massive object. Phys. Rev. X 5, 041037 (2015).

[28] Duan, L.-M., Giedke, G., Cirac, J. I. \& Zoller, P. Inseparability criterion for continuous variable systems. Phys. Rev. Lett. 84, 2722-2725 (2000).

[29] Teufel, J. D. et al. Sideband cooling of micromechanical motion to the quantum ground state. Nature 475, 359363 (2011).

[30] See Supplementary Information.

Acknowledgements We would like to thank Sorin
Paraoanu and Ian Petersen for useful discussions. This work was supported by the Academy of Finland (contracts 250280, 308290, CoE LTQ, 275245) and by the European Research Council (615755-CAVITYQPD). We acknowledge funding from the European Unions Horizon 2020 research and innovation program under grant agreement No. 732894 (FETPRO HOT). The work benefited from the facilities at the Micronova Nanofabrication Center and at the Low Temperature Laboratory infrastructure. 